\title{
A New Efficient Algorithm for the 2D WLP-FDTD Method Based on Domain Decomposition Technique
}

\author{
Bo-Ao Xu, Yan-Tao Duan, Bin Chen, Yun Yi, and Kang Luo \\ The National Key Laboratory on Electromagnetic Environmental Effects and Electro-Optical Engineering, \\ PLA University of Science and Technology, Nanjing 210007, China
}

Correspondence should be addressed to Yan-Tao Duan; dcmchdyt@126.com

Received 4 September 2016; Accepted 25 October 2016

Academic Editor: Luciano Tarricone

Copyright (C) 2016 Bo-Ao Xu et al. This is an open access article distributed under the Creative Commons Attribution License, which permits unrestricted use, distribution, and reproduction in any medium, provided the original work is properly cited.

This letter introduces a new efficient algorithm for the two-dimensional weighted Laguerre polynomials finite difference timedomain (WLP-FDTD) method based on domain decomposition scheme. By using the domain decomposition finite difference technique, the whole computational domain is decomposed into several subdomains. The conventional WLP-FDTD and the efficient WLP-FDTD methods are, respectively, used to eliminate the splitting error and speed up the calculation in different subdomains. A joint calculation scheme is presented to reduce the amount of calculation. Through our work, the iteration is not essential to obtain the accurate results. Numerical example indicates that the efficiency and accuracy are improved compared with the efficient WLP-FDTD method.

\section{Introduction}

The Laguerre-based finite difference time-domain (WLPFDTD) method [1] is widely used in solving electromagnetic problems with fine structures. As it is not constrained by the Courant-Friedrich-Levy condition, the WLP-FDTD method is unconditionally stable.

However, the WLP-FDTD method often produces a huge sparse matrix equation in the process of solving the fine structure problem. Directly solving the associate matrix is a challenging work. In order to settle this problem, He et al. introduced the domain decomposition finite difference technique to the WLP-FDTD method [2]. The huge sparse matrix is decomposed into several small ones. Duan et al. investigated an efficient WLP-FDTD method [3], in which the huge sparse matrix is turned into tridiagonal matrixes by introducing a perturbation term. CPU time and memory storage can be saved greatly. However, the splitting error caused by the perturbation term turns to be pronounced in the fine structure area. To improve this efficient method, Chen et al. studied a new splitting scheme by introducing a new perturbation term [4]. However, the iteration is required in this method, which increases the computational time.
In this letter, we introduce a new efficient algorithm for the 2D WLP-FDTD method. The whole computational domain is decomposed into several subdomains utilizing the domain decomposition scheme. In order to eliminate the splitting error, the conventional WLP-FDTD method [1] is applied to the subdomains where the fields have larger spatial derivatives. And to speed up the calculation, the efficient WLP-FDTD method [4] is used to the other subdomains where the splitting error is limited. Meanwhile, we devise a joint calculation scheme to further reduce the computational time. Through using this approach, the iteration is no longer necessary to get the accurate results. Numerical example shows that the accuracy can be improved and the CPU time is reduced to about $52.7 \%$ of the original one [4].

\section{Theories}

2.1. Domain Decomposition Technique. The domain decomposition technique is similar to that in [2]; the computational domain is decomposed into nine subdomains, as shown in Figure 1. However, in this paper, the conventional WLPFDTD method is applied to $D_{5}$ (fine structure exists). Meanwhile, the efficient WLP-FDTD method is used to the 


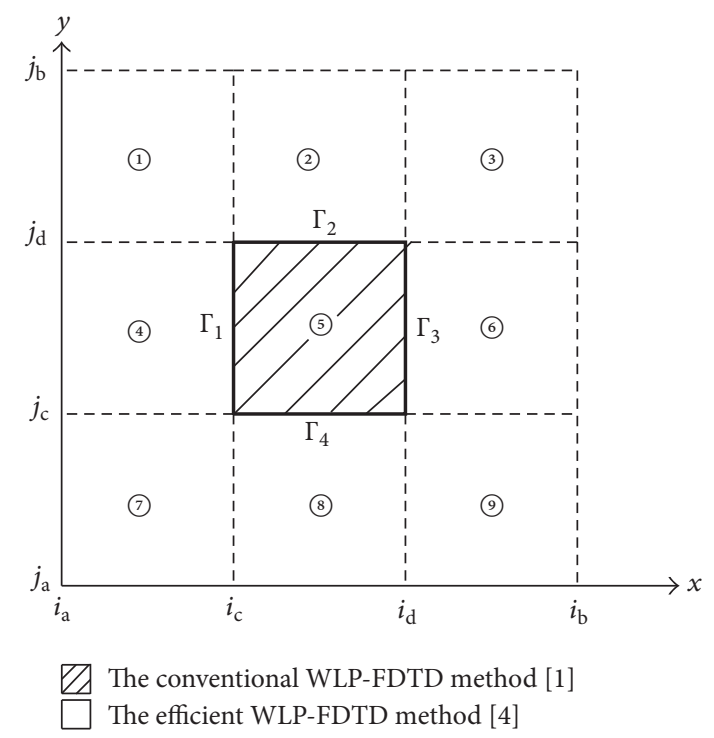

Figure 1: Nine subdomains in the computational domain.

other subdomains and the interface $\Gamma$, where $\Gamma=\Gamma_{1} \cup \Gamma_{2} \cup$ $\Gamma_{3} \cup \Gamma_{4}$.

In the simple and lossless medium, Maxwell's equations of $2 \mathrm{D} \mathrm{TE} z$ wave for the Laguerre-FDTD can be deduced as [4]

$$
\begin{aligned}
- & \left.\frac{a b}{\Delta x^{2}} E_{y}^{q}\right|_{i-1, j}+\left.\left(1+\frac{2 a b}{\Delta x^{2}}\right) E_{y}^{q}\right|_{i, j}-\left.\frac{a b}{\Delta x^{2}} E_{y}^{q}\right|_{i+1, j} \\
= & \frac{2 a b}{\Delta x \Delta y} \sum_{k=0}^{q-1}\left(\left.E_{x}^{k}\right|_{i, j+1}-\left.E_{x}^{k}\right|_{i-1, j+1}-\left.E_{x}^{k}\right|_{i, j}+\left.E_{x}^{k}\right|_{i-1, j}\right) \\
& -\left.2 \sum_{k=0}^{q-1} E_{y}^{k}\right|_{i, j}+\frac{2 a}{\Delta x} \sum_{k=0}^{q-1}\left(\left.H_{z}^{k}\right|_{i, j}-\left.H_{z}^{k}\right|_{i-1, j}\right) \\
& -\left.a J_{y}^{q}\right|_{i, j} \\
- & \left.\frac{a b}{\Delta y^{2}} E_{x}^{q}\right|_{i, j-1}+\left.\left(1+\frac{2 a b}{\Delta y^{2}}\right) E_{x}^{q}\right|_{i, j}-\left.\frac{a b}{\Delta y^{2}} E_{x}^{q}\right|_{i, j+1} \\
= & -\frac{a b}{\Delta x \Delta y}\left(\left.E_{y}^{q}\right|_{i+1, j}-\left.E_{y}^{q}\right|_{i+1, j-1}-\left.E_{y}^{q}\right|_{i, j}+\left.E_{y}^{q}\right|_{i, j-1}\right) \\
& -\left.2 \sum_{k=0}^{q-1} E_{x}^{k}\right|_{i, j}-\frac{2 a}{\Delta y} \sum_{k=0}^{q-1}\left(\left.H_{z}^{k}\right|_{i, j}-\left.H_{z}^{k}\right|_{i, j-1}\right) \\
a= & \frac{2}{s \varepsilon_{0}}, \\
b= & \frac{2}{s \mu_{0}},
\end{aligned}
$$

where $\varepsilon_{0}$ is the free space electric permittivity, $\mu_{0}$ is the free space magnetic permeability, and $\left.J_{y}^{q}\right|_{i, j}$ is the electric excitation source. The time-scale factor and order of the Laguerre polynomials are defined as $s$ and $q$, respectively.
Rewriting (1a) and (1b) as the matrix equations, we have

$$
\begin{aligned}
& {[A]\left\{y^{q}\right\}=\left\{f_{1}^{q-1}\right\}} \\
& {[B]\left\{x^{q}\right\}=\left\{f_{2}^{q-1}\right\},}
\end{aligned}
$$

where vector $x$ and $y$ represent the unknown field variables $\left.E_{x}^{q}\right|_{i, j}$ and $\left.E_{y}^{q}\right|_{i, j}$, respectively.

The conventional WLP-FDTD equations [1] can be written as

$$
[C]\left\{x y^{q}\right\}=\left\{f_{3}^{q-1}\right\},
$$

where vector $x y$ represents the unknown field variables $\left.E_{y}^{q}\right|_{i, j}$ and $\left.E_{x}^{q}\right|_{i, j}$.

According to (1a), in the subdomains $D_{1} \sim D_{3}$ and $D_{7} \sim D_{9}, A$ in (2a) correspond to the interior to interior coupling. So firstly, $y^{q}$ in these subdomains can be solved by (2a) independently. Therefore one can have the sparse matrix system as

$$
\left[\begin{array}{cccccc}
A_{44} & 0 & 0 & 0 & 0 & A_{4} \\
0 & A_{66} & 0 & 0 & 0 & A_{6} \\
0 & 0 & A_{22} & 0 & 0 & A_{2} \\
0 & 0 & 0 & A_{88} & 0 & A_{8} \\
0 & 0 & 0 & 0 & A_{55} & A_{5} \\
A_{4}^{*} & A_{6}^{*} & A_{2}^{*} & A_{8}^{*} & A_{5}^{*} & A_{\Gamma}
\end{array}\right]\left[\begin{array}{c}
y_{4} \\
y_{6} \\
x_{2} \\
x_{8} \\
x y_{5} \\
x y_{\Gamma}
\end{array}\right]=\left[\begin{array}{l}
f_{4} \\
f_{6} \\
f_{2} \\
f_{8} \\
f_{5} \\
f_{\Gamma}
\end{array}\right],
$$

where $y_{4}, y_{6}, x_{2}, x_{8}$, and $x y_{5}$, respectively, represent the field vector $y$ in $D_{4}$ and $D_{6}, x$ in $D_{2}$ and $D_{8}$, and $x y$ in $D_{5} . A_{44}$ and $A_{66}$ represent the matrix $A$ in (2a) corresponding to the interior to interior coupling in $D_{4}$ and $D_{6} . A_{22}$ and $A_{88}$ represent the matrix $B$ in (2b) corresponding to the interior to interior coupling in $D_{2}$ and $D_{8} . A_{55}$ represents the matrix $C$ in (3) corresponding to the interior to interior coupling in $D_{5}$. According to the efficient WLP-FDTD method [4], $A_{44}, A_{66}$, $A_{22}$, and $A_{88}$ are tridiagonal matrixes. The global assembly of the interior-interface and interface-interior coupling is defined as $A_{i}$ and $A_{i}^{*}$, respectively [5]. $A_{\Gamma}$ corresponds to the interface-interface coupling for $\Gamma$.

The efficient WLP-FDTD equation (1a) is applied to the interfaces $\Gamma_{1}$ and $\Gamma_{3}$; therefore the coupling terms for the vector $x\left(\Gamma_{1}\right.$ - the interior of $D_{4}$ and $\Gamma_{3}$ - the interior of $\left.D_{6}\right)$ do not exist in the sparse matrix system. However, the equations for vector $x$ in $D_{4}$ and $D_{6}$ have the coupling term of the interior-interface $\Gamma$. According to (1b), the equation for vector $x$ in $D_{147}$ and $D_{369}$ can be written as

$$
\begin{aligned}
& A_{147} x_{147}=f_{147}-B_{1} x y_{\Gamma_{1}} \\
& A_{369} x_{369}=f_{369}-B_{2} x y_{\Gamma_{3}},
\end{aligned}
$$

where $x_{147}$ and $x_{369}$ are the vector $x$ in $D_{147}$ and $D_{369} . D_{147}=$ $D_{1} \cup D_{4} \cup D_{7} ; D_{369}=D_{3} \cup D_{6} \cup D_{9} . x y_{\Gamma_{1}}$ and $x y_{\Gamma_{3}}$ are the vector $y$ in $\Gamma_{1}$ and $\Gamma_{3}$. $B_{1}$ and $B_{3}$ correspond to the interior of $D_{147}-\Gamma_{1}$ and the interior of $D_{369}-\Gamma_{3}$ coupling. 
According to (1a), the equations for vector $y$ in $D_{123}$ and $D_{789}$ can be written as

$$
\begin{aligned}
& A_{123} y_{123}=f_{123} \\
& A_{789} y_{789}=f_{789},
\end{aligned}
$$

where $D_{123}=D_{1} \cup D_{2} \cup D_{3}$ and $D_{789}=D_{7} \cup D_{8} \cup D_{9}$.

For (4), the Schur complement system is given by [6]

$$
C x y_{\Gamma}=g
$$

where

$$
\begin{gathered}
C=A_{\Gamma}-\sum_{i} A_{i}^{*} A_{i i}^{-1} A_{i}-A_{5}^{*} A_{55}^{-1} A_{5} \quad i=2,4,6,8, \\
g=f_{\Gamma}-\sum_{i} A_{i}^{*} A_{i i}^{-1} f_{i}-A_{5}^{*} A_{55}^{-1} f_{5} \quad i=2,4,6,8 .
\end{gathered}
$$

Once (7) has been solved, (4) can be solved by

$$
\begin{aligned}
A_{i i} y_{i} & =f_{i}-A_{i} x y_{\Gamma} \quad i=4,6 \\
A_{i i} x_{i} & =f_{i}-A_{i} x y_{\Gamma} \quad i=2,8 \\
A_{55} x y_{5} & =f_{5}-A_{5} x y_{\Gamma} .
\end{aligned}
$$

Then (5a) and (5b) can be solved. After that, all the calculations of $\left.E_{y}^{q}\right|_{i, j}$ and $\left.E_{x}^{q}\right|_{i, j}$ are completed.

2.2. Discussion about Reducing the Amount of Calculation. (5a), (5b), (6a), (6b), (9a), and (9b) are tridiagonal equations, so in this paper, the new algorithm has greatly decreased the amount of calculation compared with [2]. However, the matrix inversion is required in (8a) and (8b), and it is still very time consuming. In order to avoid solving the inverse matrix directly and further cut down the unnecessary calculation, we introduce a joint calculation scheme of (8a), (8b), (9a), (9b), and (9c). In this section, we take $A_{4}^{*} A_{44}^{-1} A_{4}, A_{4}^{*} A_{44}^{-1} f_{4}$, and (9a), for example.

The matrix $A_{44}$ can be written as

$$
A_{44}=\left[\begin{array}{llllll}
{\left[A_{44}\right]_{1}} & & & & & \\
& {\left[A_{44}\right]_{2}} & & & & \\
& & \ddots & & & \\
& & & {\left[A_{44}\right]_{k}} & & \\
& & & & \ddots & \\
& & & & & {\left[A_{44}\right]_{m}}
\end{array}\right] \text {, }
$$

where $m=j_{d}-j_{c}$ and $n=i_{c}-i_{a} \cdot\left[A_{44}\right]_{k}$ is a tridiagonal matrix which can be decomposed as follows:

$$
\begin{aligned}
& {\left[A_{44}\right]_{k}=L_{k} U_{k}} \\
& =\left[\begin{array}{cccccc}
l_{k, 1} & & & & & \\
a_{k, 2} & l_{k, 2} & & & & \\
& \ddots & \ddots & & & \\
& & a_{k, i} & l_{k, i} & & \\
& & & \ddots & \ddots & \\
& & & & a_{k, n} & l_{k, n}
\end{array}\right]\left[\begin{array}{cccccc}
1 & u_{k, 1} & & & & \\
& 1 & u_{k, 2} & & & \\
& & \ddots & & & \\
& & & 1 & u_{k, i} & \\
& & & \ddots & u_{k, n-1} \\
& & & & & 1
\end{array}\right] .
\end{aligned}
$$

According to (1a), $A_{4}$ and $A_{4}^{*}$ have only $m$ nonzero elements. Through some complex manipulations, one can obtain

$$
A_{4}^{*} A_{44}^{-1} A_{4}=\left[\begin{array}{cccc}
c_{1}^{*} & 0 & 0 & 0 \\
0 & 0 & 0 & 0 \\
0 & 0 & 0 & 0 \\
0 & 0 & 0 & 0
\end{array}\right] \text {, }
$$

where

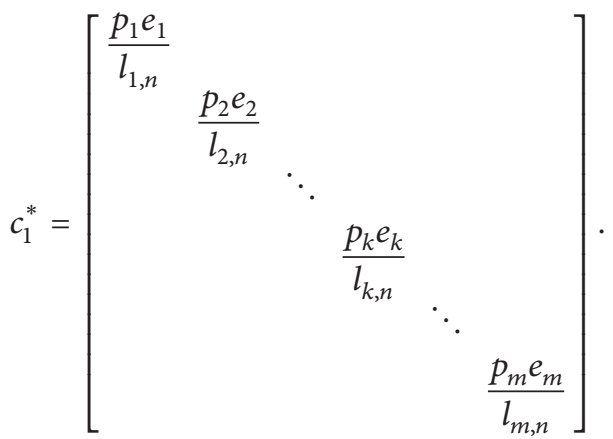

$e_{k}$ and $p_{k}$ represent the nonzero elements in $A_{4}$ and $A_{4}^{*}$ corresponding to $\left[A_{44}\right]_{k}$, respectively. (10) (12b) indicate that the calculation of $A_{4}^{*} A_{44}^{-1} A_{4}$ is quite simple and time saving due to the efficient WLP-FDTD method in $D_{4}$.

Based on (10) in $D_{4}$, (9a) can be written as

$$
\left[A_{44}\right]_{k}\left[y_{4}\right]_{k}=\left[f_{4}\right]_{k}-\left[A_{4}\right]_{k} x y_{\Gamma_{1}}=[d]_{k}
$$

Only one nonzero element $e_{k}$ is contained in $\left[A_{4}\right]_{k}$; one can have

$$
\left[A_{4}\right]_{k} x y_{\Gamma_{1}}=\left[0,0, \cdots 0, e_{k} x y_{\Gamma_{1}, k}\right]
$$

Set $\left[y_{4}^{\prime}\right]_{k}$ to meet the following equation:

$$
\left[A_{44}\right]_{k}\left[y_{4}^{\prime}\right]_{k}=\left[f_{4}\right]_{k}=\left[d^{\prime}\right]_{k}
$$

then one can obtain obviously

$$
A_{44}^{-1} f_{4}=\left[\left[y_{4}^{\prime}\right]_{1},\left[y_{4}^{\prime}\right]_{2}, \ldots\left[y_{4}^{\prime}\right]_{k}, \cdots\left[y_{4}^{\prime}\right]_{m}\right]^{\mathrm{T}} .
$$

As there is only one nonzero element $p_{k}$ in $A_{4}^{*}$ corresponding to $\left[A_{44}\right]_{k}$, one can have 
TABLE 1: Simulation results for the different methods.

\begin{tabular}{lcccc}
\hline Scheme & $\Delta t$ & Number of iterations $(q)$ & CPU time $(\mathrm{s})$ & Memory $(\mathrm{MB})$ \\
\hline WLP-FDTD & $1.0 \mathrm{ps}$ & 161 & 15.57 & 74.58 \\
Efficient WLP-FDTD [4] & $1.0 \mathrm{ps}$ & 81 & 4.7 & 1.88 \\
Proposed & $1.0 \mathrm{ps}$ & 161 & 2.48 & 3.06 \\
\hline
\end{tabular}

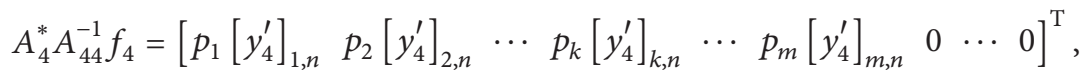

where $\left[y_{4}^{\prime}\right]_{k, n}$ is the last element of $\left[y_{4}^{\prime}\right]_{k}$ in (15).

Equations (13) and (15) are tridiagonal equations and the coefficient matrixes are all $\left[A_{44}\right]_{k}$. According to (13) (15), only the last element is different between $[d]_{k}$ and $\left[d^{\prime}\right]_{k}$

$$
\begin{aligned}
& d_{k, i}^{\prime}=d_{k, i} \quad(i=1,2, \ldots, n-1), \\
& d_{k, n}^{\prime}=d_{k, n}+e_{k} x y_{\Gamma_{1} k} .
\end{aligned}
$$

Therefore, we use the chasing method to the joint calculation of (13) and (15). The procedures are as follows:

$$
\begin{aligned}
& x_{k, i}=\frac{\left(d_{k, i}-x_{k, i-1} a_{k, i}\right)}{l_{k, i}} \quad(i=1,2, \ldots, n-1) \\
& x_{k, n}^{\prime}=\frac{\left(d_{k, n}^{\prime}-x_{k, n-1} a_{k, n}\right)}{l_{k, n}} \\
& {\left[y_{4}^{\prime}\right]_{k, n}=x_{k, n}^{\prime}} \\
& x_{k, n}=x_{k, n}^{\prime}-\frac{e_{k} x y_{\Gamma_{1} k}}{l_{k, n}} \\
& {\left[y_{4}\right]_{k, n}=x_{k, n}} \\
& {\left[y_{4}\right]_{k, i}=x_{k, i}-u_{k, i}\left[y_{4}\right]_{k, i+1}} \\
& (i=n-1, n-2, \ldots, 1) .
\end{aligned}
$$

It can be seen from $(19 a) \sim(19 c)$ that the calculation of $\left[y_{4}^{\prime}\right]_{k, n}$ is independent of $x y_{\Gamma_{1}}$. By inserting the results into (17), one can obtain $A_{4}^{*} A_{44}^{-1} f_{4}$. The calculation of $\left[y_{4}\right]_{k, n}$ is dependent on $x y_{\Gamma_{1}}$; therefore once (7) has been solved, (19d) (19f) can be solved. The method we introduced above avoids solving $A_{44}^{-1}$ directly. And due to the joint calculation scheme, in addition to (12a), (12b), (17), (19b), and (19c), the total amount of calculation of $A_{4}^{*} A_{44}^{-1} A_{4}, A_{4}^{*} A_{44}^{-1} f_{4}$, and (13) is the same as solving (13) alone. Therefore, the method has cut down the unnecessary calculation effectively. In the same way, one can deal with the other parts of $\sum_{i} A_{i}^{*} A_{i i}^{-1} A_{i}, \sum_{i} A_{i}^{*} A_{i i}^{-1} f_{i}$, $(9 a)$, and (9b).

\section{Numerical Results}

In order to validate the effectiveness of the new algorithm, a numerical example is implemented. A parallel plate waveguide with perfect electric conductor (PEC) slot of $0.2 \mathrm{~mm}$ is shown in Figure 2, the same as [4]. The PEC is infinitely thin. In order to simulate this fine structure, we take the graded mesh with an expansion factor $\alpha=1.07$ for the $y$-axis $(\alpha=$ 1.00 for the $x$-axis). In the $x$ and $y$ directions, there are 250 and 130 subdivisions including $20 \times \Delta y$ uniform cells for the PEC slot, and there are 10 and 40 subdivisions in $D_{5}$. The size of the minimum grid is $5 \mathrm{~mm} \times 10 \mu \mathrm{m}$. Mur's first-order absorbing boundary conditions [7] are set to end the $x$ boundaries. The excitation is located at the position of $(80 \Delta x, j)$ and the wave is sinusoidal modulated Gaussian pulse:

$$
J_{y}(t)=\exp \left(-\left(\frac{t-T_{c}}{T_{d}}\right)^{2}\right) \sin \left(2 \pi f_{c} t\right),
$$

where $T_{d}=1 / 2 f_{c}, T_{c}=3 T_{d}$, and $f_{c}=4 \mathrm{GHz}$. The time duration $T_{f}=4 \mathrm{~ns}, q=160$, and $s=3 \times 10^{11}[8,9]$ are chosen for the conventional WLP-FDTD and the proposed method, and $\Delta t=1.0 \mathrm{ps}$ is set to solve the Laguerre coefficient of the excitation pulse.

Figure 3 shows the vector $y$ of the electric field at the observation point. Good agreement can be found between the conventional WLP-FDTD, the efficient WLP-FDTD [4], and the proposed method.

Table 1 illustrates the CPU time for the numerical simulations. It can be observed that the CPU time for the proposed method is reduced to about $15.9 \%$ of the conventional WLPFDTD and $52.7 \%$ of the efficient WLP-FDTD. As the size of $D_{5}$ is very small, the conventional WLP-FDTD calculated in this subdomain is barely time consuming. Furthermore, the iteration is not necessary in the proposed method as the splitting error does not exist in the fine structure area. Thus the efficiency has improved.

Figure 4 depicts the error of $E_{y}$ at the observation point which is defined by $E_{y \text {, error }}=E_{y \text {, num }}-E_{y \text {,WLP-FDTD, }}$ where $E_{y, \text { num }}$ is the results solved by the efficient WLPFDTD and the proposed method, respectively. $E_{y, \text { WLP-FDTD }}$ is the solution utilizing the conventional WLP-FDTD. The accuracy of the proposed method is obviously improved compared with the original one.

However, the memory storage required in the proposed method is slightly larger than that of the efficient WLPFDTD. The conventional WLP-FDTD in $D_{5}$ whose coefficient matrix is not a tridiagonal matrix and the Schur complement system are needful in the proposed method. So the memory storage will be increased inevitably. In this letter, 


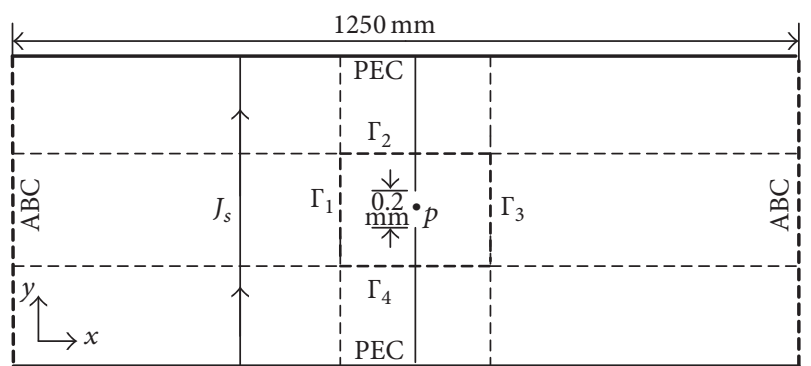

FIGURE 2: Parallel plate waveguide with the PEC slot of $0.2 \mathrm{~mm} \mathrm{[4].}$

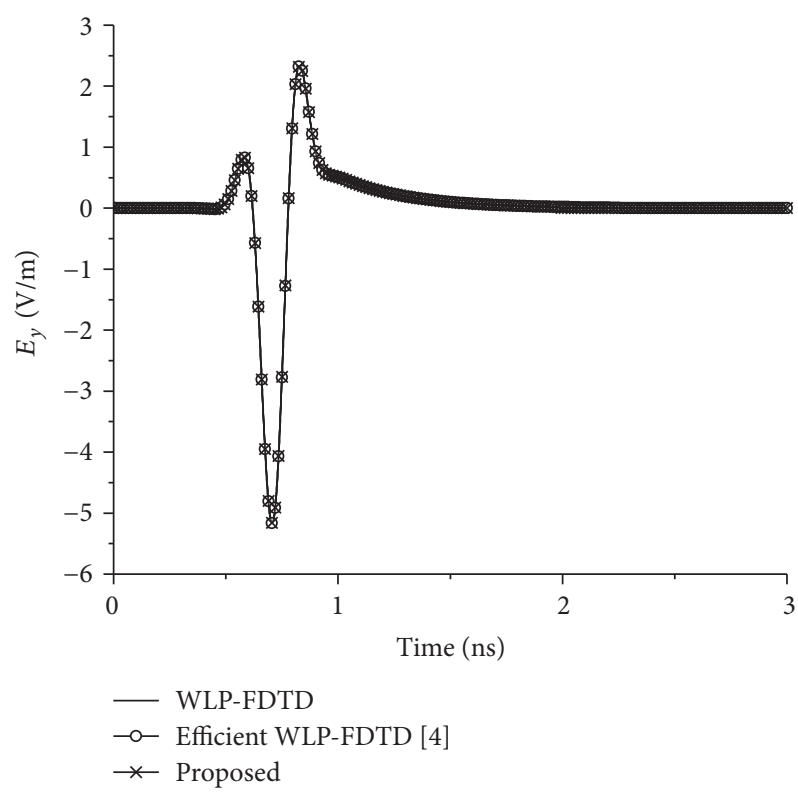

FIGURE 3: Transient electric fields of the vector $y$ at $p(100 \Delta x, 65 \Delta y)$.

all calculations have been performed on a Core 4 2.93-GHz machine.

\section{Conclusion}

In this letter, we devise a new efficient algorithm for the 2D WLP-FDTD method based on domain decomposition scheme. The whole computational domain is decomposed into several subdomains. The conventional WLP-FDTD and the efficient WLP-FDTD methods are introduced to different subdomains. And a joint calculation scheme is applied to the new algorithm. Compared with the efficient WLP-FDTD, the proposed algorithm does not need any iteration. Numerical example has verified that the proposed method can save CPU time and improve the computational accuracy. The research for the $3 \mathrm{D}$ case will be presented in the further work.

\section{Competing Interests}

The authors declare that they have no competing interests.

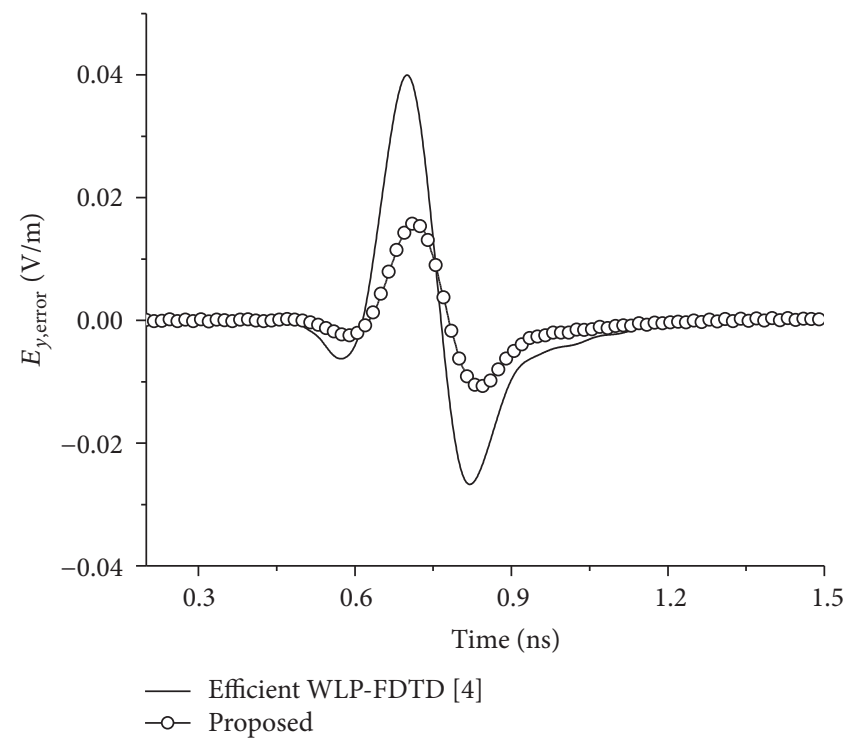

FIgURE 4: Error of $E_{y}$ at $p(100 \Delta x, 65 \Delta y)$. Calculated by the proposed method and the efficient WLP-FDTD [4].

\section{Acknowledgments}

This work was supported in part by the National Science Foundation of China under Grant 51477182.

\section{References}

[1] Y.-S. Chung, T. K. Sarkar, B. H. Jung, and M. Salazar-Palma, "An unconditionally stable scheme for the finite-difference timedomain method," IEEE Transactions on Microwave Theory and Techniques, vol. 51, no. 3, pp. 697-704, 2003.

[2] G.-Q. He, W. Shao, X.-H. Wang, and B.-Z. Wang, "An efficient domain decomposition Laguerre-FDTD method for twodimensional scattering problems," IEEE Transactions on Antennas and Propagation, vol. 61, no. 5, pp. 2639-2645, 2013.

[3] Y.-T. Duan, B. Chen, and Y. Yi, "Efficient implementation for the unconditionally stable 2-D WLP-FDTD method," IEEE Microwave and Wireless Components Letters, vol. 19, no. 11, pp. 677679, 2009.

[4] Z. Chen, Y.-T. Duan, Y.-R. Zhang, and Y. Yi, "A new efficient algorithm for the unconditionally stable 2-D WLP-FDTD method," IEEE Transactions on Antennas and Propagation, vol. 61, no. 7, pp. 3712-3720, 2013.

[5] B.-Z. Wang, R. Mittra, and W. Shao, "A domain decomposition finite-difference method utilizing characteristic basis functions for solving electrostatic problems," IEEE Transactions on Electromagnetic Compatibility, vol. 50, no. 4, pp. 946-952, 2008.

[6] T. N. Phillips, "Preconditioned iterative methods for elliptic problems on decomposed domains," International Journal of Computer Mathematics, vol. 44, no. 1-4, pp. 5-18, 1992.

[7] G. Mur, "Absorbing boundary conditions for the finite-difference approximation of the time-domain electromagnetic-field equations," IEEE Transactions on Electromagnetic Compatibility, vol. 23, no. 4, pp. 377-382, 1981.

[8] Z. Mei, Y. Zhang, X. Zhao, B. H. Jung, T. K. Sarkar, and M. Salazar-Palma, "Choice of the scaling factor in a marchingon-in-degree time domain technique based on the associated 
Laguerre functions," IEEE Transactions on Antennas and Propagation, vol. 60, no. 9, pp. 4463-4467, 2012.

[9] M. Yuan, J. Koh, T. K. Sarkar, W. Lee, and M. Salazar-Palma, "A comparison of performance of three orthogonal polynomials in extraction of wide-band response using early time and low frequency data," IEEE Transactions on Antennas and Propagation, vol. 53, no. 2, pp. 785-792, 2005. 


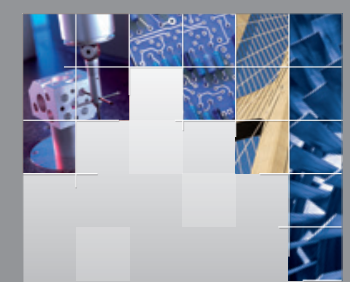

\section{Enfincering}
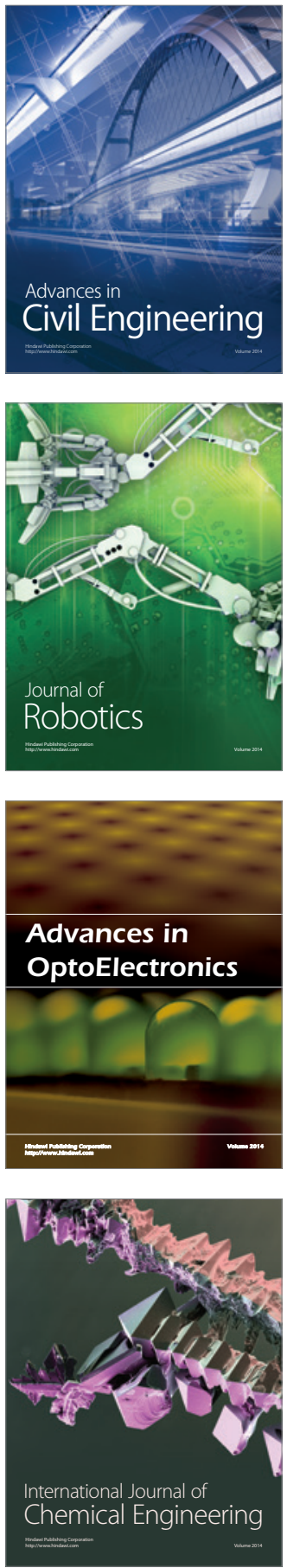

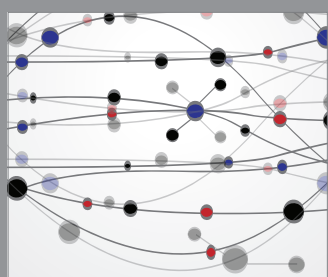

The Scientific World Journal

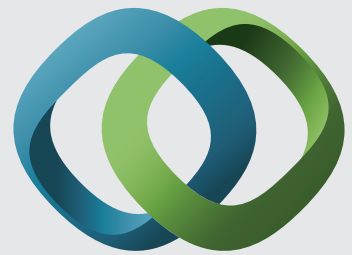

\section{Hindawi}

Submit your manuscripts at

http://www.hindawi.com
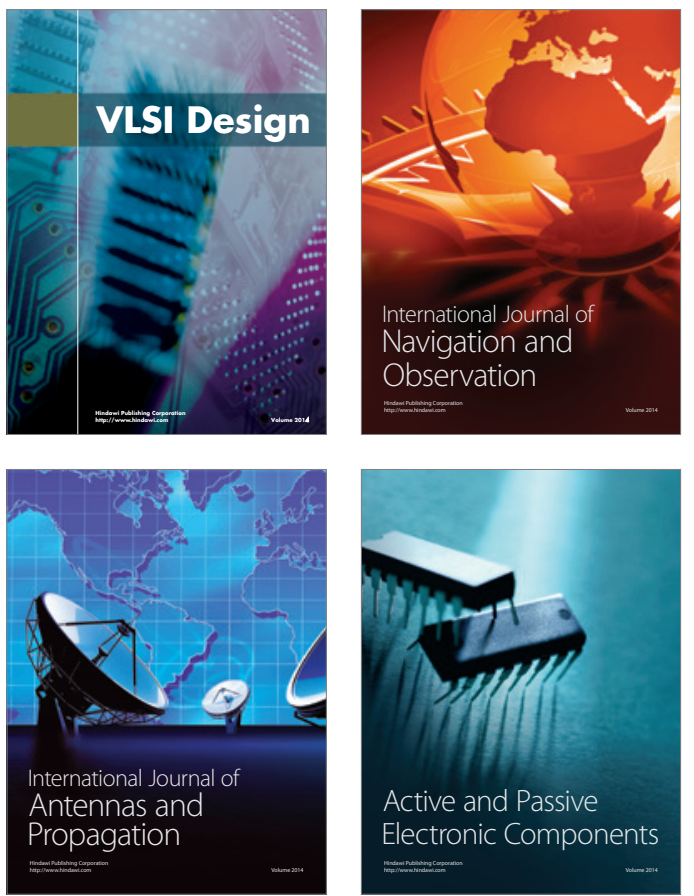
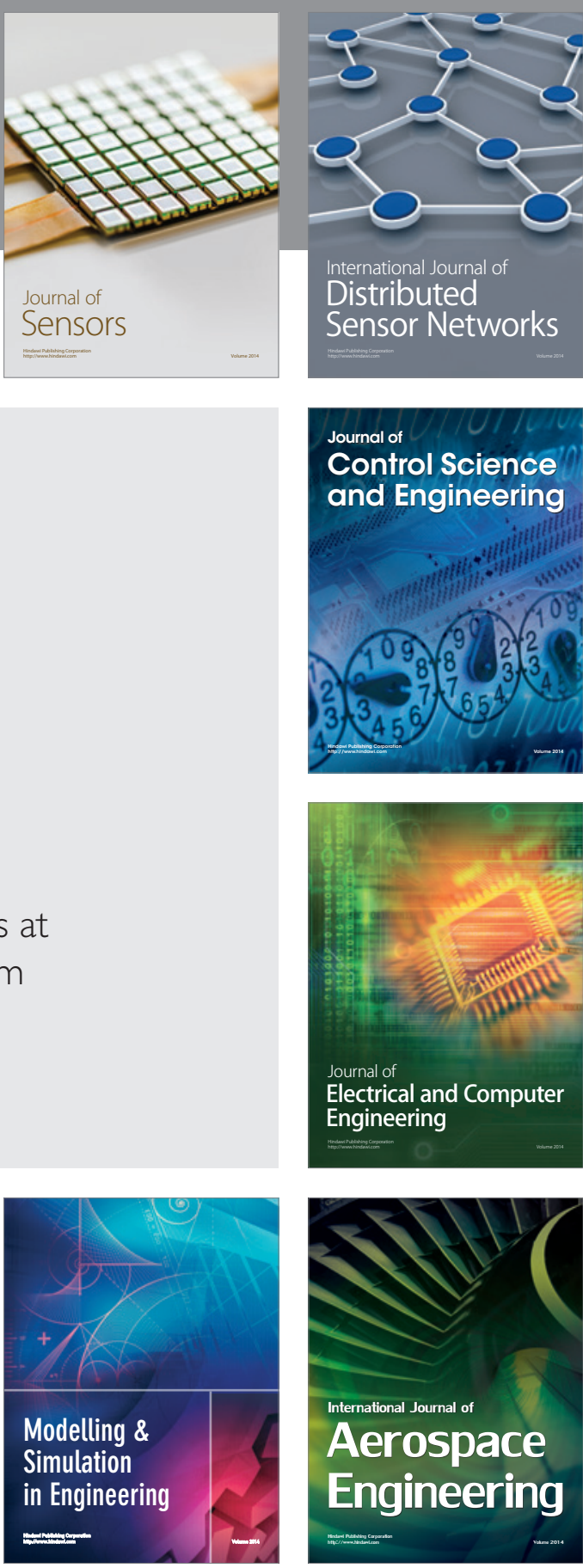

International Journal of

Distributed

Sensor Networks

Journal of

Control Science

and Engineering
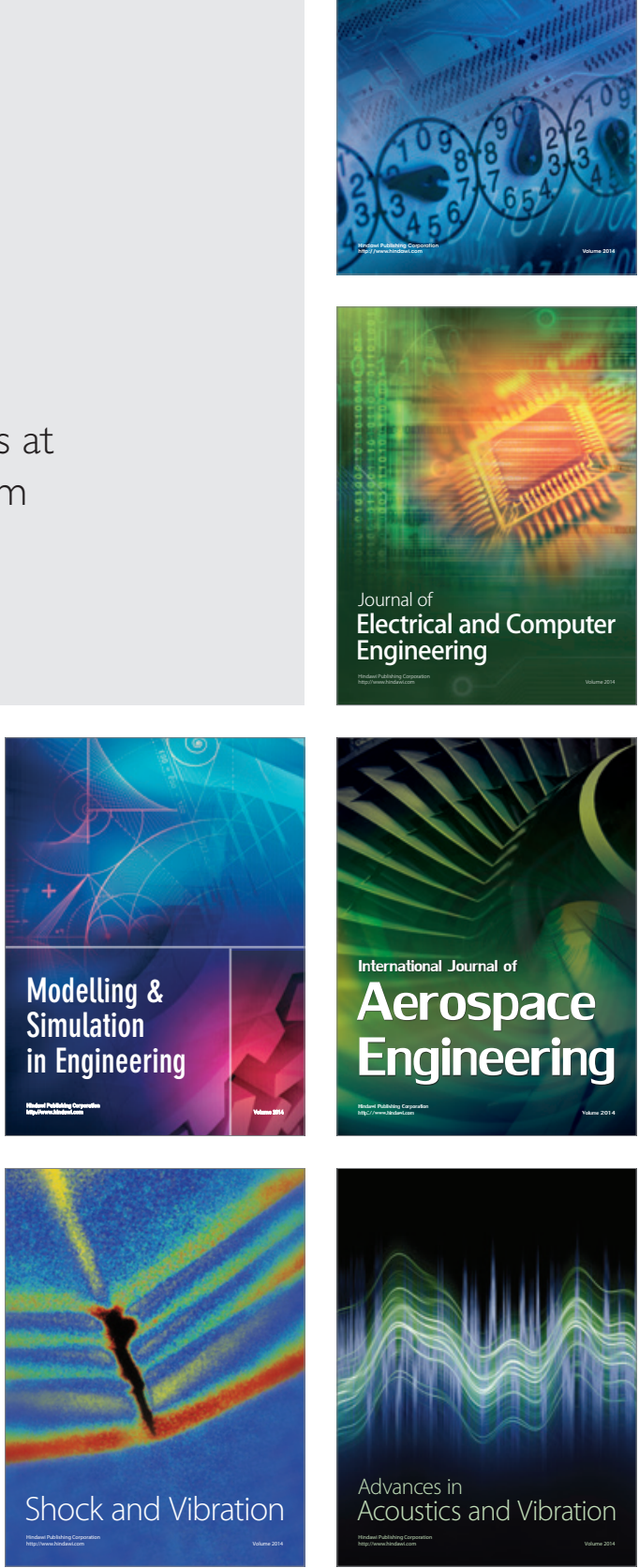